Nature. 2006 April 6; 440(7085): . doi:10.1038/nature04642.

\title{
Crystal structure of the CorA $\mathrm{Mg}^{2+}$ transporter
}

\author{
Vladimir V. Lunin ${ }^{1,{ }^{*}}$, Elena Dobrovetsky ${ }^{2,{ }^{*}}$, Galina Khutoreskaya ${ }^{2}$, Rongguang Zhang ${ }^{4}$, \\ Andrzej Joachimiak ${ }^{4}$, Declan A. Doyle ${ }^{5}$, Alexey Bochkarev ${ }^{2,3,6}$, Michael E. Maguire ${ }^{7}$, Aled \\ M. Edwards $\mathbf{s}^{1,2,3,4,6}$, and Christopher M. Koth ${ }^{2, \dagger}$ \\ ${ }^{1}$ Department of Medical Biophysics, University of Toronto, 112 College Street, Toronto, Ontario \\ M5G 1L6, Canada \\ ${ }^{2}$ Banting and Best Department of Medical Research, University of Toronto, 112 College Street, \\ Toronto, Ontario M5G 1L6, Canada \\ ${ }^{3}$ Department of Medical Genetics and Microbiology, University of Toronto, 112 College Street, \\ Toronto, Ontario M5G 1L6, Canada \\ ${ }^{4}$ Structural Biology Center and Midwest Center for Structural Genomics, Biosciences Division, \\ Argonne National Laboratory, 9700 S. Cass Avenue, Argonne, Illinois 60439, USA \\ ${ }^{5}$ Structural Genomics Consortium, Botnar Research Centre, Oxford, Oxon OX3 7LD, UK \\ ${ }^{6}$ Structural Genomics Consortium, Banting Institute, 100 College Street, Toronto, Ontario M5G \\ 1L5, Canada \\ ${ }^{7}$ Department of Pharmacology, Case Western Reserve University, Cleveland, Ohio 44106-4965, \\ USA
}

\section{Abstract}

The magnesium ion, $\mathrm{Mg}^{2+}$, is essential for myriad biochemical processes and remains the only major biological ion whose transport mechanisms remain unknown. The CorA family of magnesium transporters is the primary $\mathrm{Mg}^{2+}$ uptake system of most prokaryotes ${ }^{1-3}$ and a functional homologue of the eukaryotic mitochondrial magnesium transporter ${ }^{4}$. Here we determine crystal structures of the full-length Thermotoga maritima CorA in an apparent closed state and its isolated cytoplasmic domain at $3.9 \AA$ and $1.85 \AA$ resolution, respectively. The transporter is a funnel-shaped homopentamer with two transmembrane helices per monomer. The channel is formed by an inner group of five helices and putatively gated by bulky hydrophobic residues. The large cytoplasmic domain forms a funnel whose wide mouth points into the cell and whose walls are formed by five long helices that are extensions of the transmembrane helices. The cytoplasmic neck of the pore is surrounded, on the outside of the funnel, by a ring of highly conserved positively charged residues. Two negatively charged helices in the cytoplasmic domain extend back towards the membrane on the outside of the funnel and abut the ring of positive charge. An apparent $\mathrm{Mg}^{2+}$ ion was bound between monomers at a conserved site in the cytoplasmic domain, suggesting a mechanism to link gating of the pore to the intra-cellular concentration of $\mathrm{Mg}^{2+}$.

\section{(C) 2006 Nature Publishing Group}

Correspondence and requests for materials should be addressed to A.M.E. (aled.edwards@utoronto.ca) or M.E.M. (mem6@cwru.edu). These authors contributed equally to this work.

†Present address: Vertex Pharmaceuticals Incorporated, 130 Waverly Street, Cambridge, Massachusetts 02139, USA

Author Information: Reprints and permissions information is available at npg.nature.com/reprintsandpermissions. The authors declare no competing financial interests.

Supplementary Information is linked to the online version of the paper at www.nature.com/nature. 
The CorA magnesium transporter is a homopentamer with fivefold symmetry about a central pore and can be divided into three parts (Fig. 1). A carboxy-terminal transmembrane domain comprises two transmembrane helices from each monomer (Fig. 2). The middle portion resembles a funnel, narrow at the entrance $(\sim 5 \AA)$ and wide at the mouth $(\sim 20 \AA)$, that is formed largely by a long a-helix extension of the inner transmembrane helix. Finally, a large cytoplasmic domain lies exterior to the funnel.

The cytoplasmic domain of CorA is a seven-stranded parallel/antiparallel $\beta$-sheet

$(\beta 2 \Delta \beta 1 \nabla \beta 3 \Delta \beta 7 \Delta \beta 6 \nabla \beta 5 \Delta \beta 4 \nabla)$ sandwiched between two sets of a-helices (a1, $\alpha 2, \alpha 3)$ and $(\alpha 4, a 5, a 6)$ (Fig. 1). The domain fold is unlike all other known structures of ion channels or transporters and constitutes a new protein fold (see Supplementary Information). This domain, solved in its soluble form at $1.85 \AA$ A resolution (Supplementary Fig. S1), is linked to the transmembrane helices by the long a7 helix (residues 251-312), termed the stalk helix. The stalk helix kinks as it enters the membrane, extends through the membrane, forms the first transmembrane helix (TM1; residues 293-312) and harbours the 'YGMNF' signature sequence of CorA (residues 311-315) ${ }^{5,6}$ (Fig. 2 and Supplementary Fig. S2). The five TM1 helices (residues 293-312) form the pore. After a short extracellular seven-amino-acid loop, the TM2 helix (residues 326-345) returns back to the cytoplasm and ends in a highly conserved C-terminal KKKKWL motif (Fig. 3). In the current structure, neither the extracellular loop nor the final two amino acids could be resolved.

The cytoplasmic domain shows the lowest sequence conservation among CorA transporters. Nonetheless, the predicted secondary structure of CorA orthologues agrees well with the three-dimensional structure of the T. maritima CorA, allowing the prediction of the structure of both the prokaryotic $\mathrm{ZntB} \mathrm{Zn}^{2+}$ efflux transporter ${ }^{7}$ and eukaryotic mitochondrial MRS2 homologues ${ }^{8}$ as CorA-like structures even though these proteins have minimal sequence conservation with CorA (Supplementary Fig. S3).

The transmembrane region comprises two concentric rings of helices. The TM2 helices, which are essentially perpendicular to the membrane, form an outer ring that envelops the inner ring of TM1 helices, which form the pore. The TM1 helices are twisted along the fivefold axis and kinked in two places, at Pro 303 and Gly 312 (Fig. 2), resulting in a tapering of the pore near the periplasmic surface. Pro 303 is conserved in a similar position in almost all CorA orthologues. The distortion of transmembrane helices by proline or glycine residues is a feature noted in other ion channels and can provide flexibility during the dynamic gating process $^{9,10}$.

The CorA structure appears to represent a closed form of the transporter. A series of constrictions are present along the transmembrane section of the ion conduction pathway. These start with a ring of five Asn 314 side chains at the periplasmic entrance that probably block ion movement (Figs 2 and 4). The Asn 314 ring appears to be held in conformation, at least in part, by an aromatic stacking interaction between Tyr311 of one monomer and Phe 315 of an adjacent monomer (that is, $\mathrm{Y}$ and $\mathrm{F}$ of the conserved YGMNF sequence) (Fig.2 and Supplementary Fig. S2). Immediately following this sequence is a loop connecting TM1 and TM2. The loop is poorly ordered in this structure (residues 316-322 are unassigned). Nevertheless, this sequence always contains significant negative charge in those CorA family members known to transport $\mathrm{Mg}^{2+}$ (Supplementary Fig. S4) ${ }^{6,11}$. The presence of the negatively charged loop on the periplasmic face of these $\mathrm{Mg}^{2+}$ transporters strongly suggests that this region comprises part of a selectivity filter.

The cytosolic part of the transporter, shaped like a funnel, is formed by the amino-terminal domains. The five $\mathbf{a} 7$ stalk helices form both the wall of the pore (TM1) and the wall of the funnel (Fig. 1). The inner wall of the funnel is lined along its length with successive rings of 
negative or hydroxyl-bearing residues (Tyr 255, Asp 263, Glu 266, Asp 270, Ser 273, Asp 277, Ser 281, Ser 284, Glu 230, Ser 233, Asp 238, Glu 246) (Fig. 3). Such an arrangement of charged residues is found in other cation channels and may serve as an electrostatic sink to increase the local ion concentration ${ }^{12}$.

The orientation of the outer TM2 helices positions the short, highly basic $\mathrm{C}$ terminus of the protein (KKKKWL) on the intracellular side of the membrane. Given the pentameric nature of CorA, this places at least 20 lysines at the membrane interface, creating a ring of positive charge approximately $50 \AA$ in diameter, which we term the basic sphincter (Fig. 3).

Additional lysines from the $\alpha 7$ stalk helix (Lys 292 and Lys 286) may also contribute to this remarkable concentration of positive charge. Abutting the basic sphincter is a cluster of negatively charged residues from two helices in the soluble domain ( $a 5$ and a6; residues 166-202 and 208-238). These helices, which are oriented perpendicularly towards the membrane interface and 'hang' towards the membrane like the branches of a weeping willow tree, are referred to as the willow helices. Almost half the residues in the willow helices and their intervening loop are aspartate or glutamate, and are highly conserved (Fig. 3 and Supplementary Fig. S5).

$\mathrm{An} \mathrm{Mg}^{2+}$ ion entering the cell through CorA would traverse a distance of at least $40 \AA$, extending through the putative periplasmic selectivity filter, transmembrane domain and part of the cytoplasmic domain (Fig. 4). The diameter of the pathway varies considerably along its length, producing two small cavities with diameters of $\sim 6 \AA$ and $\sim 5.6 \AA$; these intersperse three constrictions. Starting from the periplasmic side, the first constriction is formed by the Asn 314 side chains. The second is formed by Thr 305 and Met 302, and the third, positioned at the membrane/cytoplasmic interface, is formed by two hydrophobic residues, Leu 294 and Met 291. There is experimental electron density inside the two cavities near Gly 309 and Ala 298 that is not readily assignable to backbone or side chain groups, consistent with the presence of $\mathrm{Mg}^{2+}$ ions. Potassium channels have a single, larger cavity, located at the centre of the bilayer, which helps to stabilize an ion ${ }^{13}$. The two cavities possibly have an analogous role for CorA. The basic sphincter is at the same level in the protein as the last constricting hydrophobic girdle. This suggests that the concentration of positive charge could be important for controlling pore diameter at this point in TM1.

Given the varying diameter throughout the pore, it appears that the transporter is in a closed state. The hydrophobic ring created by residues Leu 294 and Met 291 would create a strong energetic barrier to ion permeation and therefore be expected to form the main gate. A bulky hydrophobic residue at position 294 is a conserved feature of CorA transporters. This hydrophobic gate seems to be one of a number of recurring themes in cation selective channels $^{12,14}$ by which ion movement is controlled indirectly through the controlled movement of water. This has been observed in the closed-state structures of the potassium channel KirBac1.1 $1^{15}$, the mechanosensitive stretch receptor $\mathrm{MscL}^{16}$ and the nicotinic acetylcholine receptor $(\mathrm{nAChR})^{17}$.

Even though CorA has several structural themes in common with ion channels it transports ions much more slowly. The $\mathrm{nAChR}$ allows 100,000 ions per second through whereas $\mathrm{K}^{+}$ channels allow up to 100 million ions through per second (ref. 18). The throughput of CorA is only of the order of 10 million ions per minute for the whole cell ${ }^{3}$. This must be related to the way in which the $\mathrm{Mg}^{2+}$ ion interacts with the transporter. The problem may be that $\mathrm{Mg}^{2+}$ prefers to be hexacoordinated and probably remains so throughout the conduction process ${ }^{19}$. Hence, as the hydrated $\mathrm{Mg}^{2+}$ ion enters the conduction pathway, the number of available coordination sites would not match the five-fold symmetry of the CorA selectivity filter. The key to $\mathrm{K}^{+}$channel selectivity and throughput is the high degree to which the potassium channel's selectivity filter mimics the hydration coordination of potassium ions ${ }^{13,14}$. Hence, 
the hydrated $\mathrm{Mg}^{2+} /$ CorA symmetry mismatch may be a form of control that slows down the rate at which the $\mathrm{Mg}^{2+}$ ion can move through CorA.

The selectivity determinants of T. maritima CorA for $\mathrm{Mg}^{2+}$ are unclear because part of the periplasmic entrance to the pore, which presumably contains an ion selectivity filter, was not seen in the structure. However, the visible structural features of the CorA transporter, as well as the unique chemistry of $\mathrm{Mg}^{2+}$, limit the mechanisms by which it can be highly selective for the ion. Sieving for hydrated cations is an insufficient explanation because the hydrated radius of magnesium is the largest of all biological cations $(\sim 5 \AA)^{19}$. Based on size alone, $\mathrm{Ca}^{2+}$ would be predicted to be transported, but it is neither a substrate nor an inhibitor of CorA $\mathrm{A}^{3,20}$. Moreover, cobalt(III) hexaammine, which has the same size as a hydrated $\mathrm{Mg}^{2+}$ but carries a +3 charge, potently inhibits transport via CorA transporters ${ }^{21}$. Thus, the CorA transporter must make use of other properties of ions, in addition to their charge and hydrated size.

For several reasons, we believe that an incoming magnesium ion is at least partially dehydrated upon entering the ion conduction pore of CorA, and that this is a key basis for selectivity. First, CorA transporters can also transport $\mathrm{Co}^{2+}$ and $\mathrm{Ni}^{2+}$, two ions with atomic radii similar to that of $\mathrm{Mg}^{2+}$, but not $\mathrm{Mn}^{2+}$, whose atomic radius is significantly larger than $\mathrm{Mg}^{2+}$ (refs 3, 20). Second, the atomic $\mathrm{Mg}^{2+}$ ion is the smallest of all biological cations, $\sim 350$ times smaller than its hydrated form ${ }^{19}$. A conduction pathway that exploited the small size of the dehydrated $\mathrm{Mg}^{2+}$ ion would conceivably be highly selective. Third, the inhibitory action of cobalt (III) hexaammine, which is almost exactly the same size as a hydrated $\mathrm{Mg}^{2+}$, suggests that a fully hydrated $\mathrm{Mg}^{2+}$ ion first binds to the CorA transporter but cannot enter the pore. Fourth, the lining of the periplasmic entrance to the pore with polar groups (Figs 2, 4 and Supplementary Fig. S2) may serve to compensate for the energetic cost of dehydration. The Asn 314 constricting ring, which obviously cannot block the pore in an open conformation, may have a role in the initial $\mathrm{Mg}^{2+}$ dehydration, as occurs in other ion channels ${ }^{12,14}$.

The crystal structure of the isolated cytoplasmic domain at $1.85 \AA$ A resolution revealed electron density hexacoordinated to oxygen atoms (one from Asp 89 and five from water molecules) that can be reasonably interpreted as a $\mathrm{Mg}^{2+}$ ion (Fig. 5a and see Supplementary Information). The structure of the full-length CorA, although at lower resolution, revealed strong electron density at a similar location, between the cytoplasmic domains of adjacent monomers (Fig. 5b). This density was found proximal to residue Asp89 in the a3 helix of one monomer and Asp 253 on the stalk helix (a7) of the adjacent monomer. Asp89 and Asp 253 are among the most highly conserved residues, pointing to a critical functional role. We suggest that this putative $\mathrm{Mg}^{2+}$-binding site could be the pivot point of a hinge comprising the stalk helix and the $\alpha-\beta-\alpha$ sandwich domain, allowing metal binding to influence the orientation of these elements within the CorA pentamer. Small changes in the structure of the $\mathrm{Mg}^{2+}$-binding site might be predicted to translate into large movements of both the stalk helix and the willow helices at their opposite ends near the pore.

How might the binding of $\mathrm{Mg}^{2+}$ be exploited for regulation? CorA must have a mechanism to regulate pore opening in response to changes in the intracellular concentration of ion. It is noteworthy that the narrowest part of the pore, the putative Leu 294/Met 291 gate, is precisely at the level of the basic sphincter noted above. The sphincter might be predicted to draw negative charge away from the middle of the pore at this level, preventing passage of the positively charged $\mathrm{Mg}^{2+}$ cation. The presence of a putative $\mathrm{Mg}^{2+}$-binding site in the soluble domain links important structural elements within the transporter and suggests a mechanism to open the gate. In the presence of sufficient magnesium, the $\alpha-\beta-\alpha$ domain would be kept near to the stalk helix of the adjacent monomer, pulling the willow helices 
away from the membrane and the basic sphincter residues apparently closing the pore. Loss of $\mathrm{Mg}^{2+}$ from the binding site between $\alpha 7$ of one monomer and $\alpha 3$ of the adjacent monomer would allow rotation or other movement of the $\alpha-\beta-\alpha$ domain. This would necessarily cause movement of the $\alpha 7$ stalk helix and of the acidic willow helices relative to the basic sphincter. Because the membraneproximal portions of the willow helices are highly negatively charged, this would in turn influence the effective concentration of positive charge created by the basic sphincter, perhaps causing it to relax. Coupled with movement of the extremely long a7 helix, this would allow the gate formed by Leu 294/Met 291 to open, allowing $\mathrm{Mg}^{2+}$ through.

Key residues within the pore-forming TM1 domain can be interpreted with regards to Salmonella enterica serovar Typhimurium mutagenesis data ${ }^{22}$. Leu294 appears to block the pore near the cytoplasmic face. The equivalent residue in $S$. Typhimurium is Phe 259, an equally bulky residue. Mutation of the adjacent Ser 260 residue in $S$. Typhimurium shows that substitution of smaller (Ala) but not larger (Val) residues allows transport, a result compatible with Leu294/Phe 259 forming a moveable barrier for transport. Mutation of Pro 268 and Pro 269 in S. Typhimurium reduces transport, compatible with Pro 303 in $T$. maritima forming a kink and functional hinge in the TM1 helix. Mutations within the signature YGMNF sequence (residues 311-315 in T. maritima and 276-280 in S.

Typhimurium) almost invariably abolish transport. This suggests that this sequence is critical both for positioning the periplasmic loop that is presumably responsible for initial binding of $\mathrm{Mg}^{2+}$, and that Asn 314 is possibly required to restrict the external entrance to the pore. Moreover, of the multiple mutations made in this sequence in $S$. Typhimurium, Tyr 311 is the only residue that can be changed with retention of activity. In the structure of CorA, the side chain of Tyr311is pointed away from the pore, and appears to form an aromatic stacking interaction with Phe 315 of an adjacent monomer. Mutation of Tyr 311 to tryptophan, which would probably retain this interaction, has only modest effects on activity.

We have crystallized the $T$. maritima CorA $\mathrm{Mg}^{2+}$ transporter in an apparently closed state. Like the MscL mechanosensitive ion channel ${ }^{16}$, glycine ${ }^{23}$, acetylcholine ${ }^{17}, \mathrm{GABA}_{\mathrm{A}}$ and serotonin $\left(5 \mathrm{HT}_{3}\right)$ receptors ${ }^{24}$, the CorA transporter is assembled as a pentamer. However, beyond this common pentameric organization, the CorA transporter is quite different. On the basis of this structure, we propose that several key principles underlie the mechanism of $\mathrm{Mg}^{2+}$ transport through CorA. Initial selectivity occurs at the periplasmic surface and involves dehydration. In the absence of sufficient intracellular $\mathrm{Mg}^{2+}$ levels, $\mathrm{Mg}^{2+}$ ions bound between monomers are released. This causes the willow helices to shift and the basic sphincter, which is in register with the Leu 294/Met 291 gate, to open and allow $\mathrm{Mg}^{2+}$ ions to flow through. In addition to the Leu 294/Met 291 gate and the basic sphincter, other regions of CorA probably widen to permit ion transport. In short, selectivity of CorA transporters is achieved at the periplasmic face, but intracellular $\mathrm{Mg}^{2+}$ determines transport. Clearly, support for this mechanism will require further functional studies. Nevertheless, the structure of T. maritima CorA should serve as an attractive model for understanding the mechanisms regulating $\mathrm{Mg}^{2+}$ ion transport in this broad protein family.

\section{Methods}

\section{Expression of full-length and soluble domain of T. maritima CorA}

The coding sequence of T. maritima CorA and its $\mathrm{N}$-terminal domain (residues 1-266) were expressed in Escherichia coli as fusions to an N-terminal sixhistidine tag in a modified T7 polymerase expression vector. After growth of the cells and induction of protein expression, the cells were harvested, resuspended, lysed and the membrane fraction isolated. CorA was solubilized from this fraction in a solution containing 50mM HEPES pH7.5, 500mM NaCl, 
$5 \%$ glycerol, $10 \mathrm{mM}$ imidazole, $1 \% \mathrm{n}$-dodecyl- $\beta$-D-maltopyranoside (DDM, Anatrace) and protease inhibitors and then purified by nickel affinity chromatography. Expression of selenomethionine-labelled proteins was essentially as described 25 .

Crystals of full-length T. maritima CorA and the CorA soluble domain were grown in hanging drops over a solution containing either 20\% (w/v) PEG 2000 (Fluka), $0.3 \mathrm{M}$ $\mathrm{Mg}\left(\mathrm{NO}_{3}\right)_{2}$ and $0.1 \mathrm{M}$ Tris pH 8.0, or 35\% (w/v) PEG 3350 (Fluka), $0.2 \mathrm{M} \mathrm{MgCl}$ and $0.1 \mathrm{M}$ Tris $\mathrm{pH} 8.5$, respectively. Detailed purification and crystallization procedures can be found at http://sgc.utoronto.ca/SGC-WebPages/StructureDescription/2BBJ.php and in the Supplementary Information.

\section{Structure determination}

Native crystals of full-length CorA diffracted X-rays to $3.9 \AA$ A Selenomethionine-containing crystals formed only from protein preparations that had low incorporation $(<20 \%)$ of selenomethionine. The low occupancy prevented us from solving the selenium substructure from these data. Phase information for the crystals of the full-length protein was obtained by first determining the $1.85 \AA$ structure of the amino-terminal two-thirds of CorA (residues 1244) and using this structure as a search model for molecular replacement.

The final model of the N-terminal domain of CorA contained residues 13-117, 120-199 and 207-244, 236 water molecules and two metal ions, assigned as $\mathrm{Mg}^{2+}$ and $\mathrm{Na}^{+}$, and four molecules of detergent (DDM). The model for the full-length protein, derived from the molecular replacement solution, was built initially by fitting the $\mathrm{N}$-terminal domain into the electron density and then improved by a combination of building, refining and five-fold noncrystallographic averaging. To help to register the amino acid sequence within the mostly helical electron density, phases taken from this improved model were used to calculate an anomalous Fourier map from the data collected from selenomethioninecontaining crystals. Inspection of this map revealed eight peaks and their noncrystallographic symmetry mates; three peaks corresponded exactly to the positions of the Met residues in the soluble domain. There were two additional peaks inside the pore and three outside the pore. The register of the assigned sequence based on the conveniently placed methionine signposts corresponds well with two kinks in the stalk helix at positions Gly 274 and Pro 303. The final model, which was refined to $3.9 \AA$, contains residues 9-315 and 323-349. The $R$ factor was 0.361 , and $R_{\text {free }}=0.406$.

\section{Supplementary Material}

Refer to Web version on PubMed Central for supplementary material.

\section{Acknowledgments}

The research was supported by grants from Genome Canada, the National Institutes of Health, the US Department of Energy, Office of Biological and Environmental Research, and the Structural Genomics Consortium. We thank M. McMillan and Y. Kim for assistance in collecting data, and A. Savchenko and the Indians and Red Wings screening teams for their guidance. We also thank C. Charky and D. Bouchard at Nextal Biotechnologies for their assistance throughout our membrane protein crystallization efforts.

\section{References}

1. Nelson DL, Kennedy EP. Magnesium transport in Escherichia coli Inhibition by cobaltous ion. J Biol Chem. 1971; 246:3042-3049. [PubMed: 4928897]

2. Hmiel SP, Snavely MD, Miller CG, Maguire ME. Magnesium transport in Salmonella typhimurium: Characterization of magnesium influx and cloning of a transport gene. J Bacteriol. 1986; 168:14441450. [PubMed: 3536881] 
3. Bui DM, Gregan J, Jarosch E, Ragnini A, Schweyen RJ. The bacterial magnesium transporter CorA can functionally substitute for its putative homologue Mrs2p in the yeast inner mitochondrial membrane. J Biol Chem. 1999; 274:20438-20443. [PubMed: 10400670]

4. Kehres DG, Maguire ME. Structure, properties and regulation of magnesium transport proteins. Biometals. 2002; 15:261-270. [PubMed: 12206392]

5. Kehres DG, Lawyer CH, Maguire ME. The CorA magnesium transporter gene family. Microb Compar Genom. 1998; 43:151-169.

6. Worlock AJ, Smith RL. ZntB is a novel $\mathrm{Zn}^{2+}$ transporter in Salmonella enterica serovar Typhimurium. J Bacteriol. 2002; 184:4369-4373. [PubMed: 12142406]

7. Gardner RC. Genes for magnesium transport. Curr Opin Plant Biol. 2003; 6:263-267. [PubMed: 12753976]

8. Cordes FS, Bright JN, Sansom MS. Proline-induced distortions of transmembrane helices. J Mol Biol. 2002; 323:951-960. [PubMed: 12417206]

9. Tieleman DP, Shrivastava IH, Ulmschneider MR, Sansom MS. Proline-induced hinges in transmembrane helices: possible roles in ion channel gating. Proteins. 2001; 44:63-72. [PubMed: 11391769]

10. Knoop V, Groth-Malonek M, Gebert M, Eifler K, Weyand K. Transport of magnesium and other divalent cations: evolution of the 2-TM-GxN proteins in the MIT superfamily. Mol Genet Genom. 2005; 274:205-216.

11. Doyle DA. Molecular insights into ion channel function. Mol Membr Biol. 2004; 21:221-225. [PubMed: 15371011]

12. Roux B, MacKinnon R. The cavity and pore helices in the KcsA K ${ }^{+}$channel: electrostatic stabilization of monovalent cations. Science. 1999; 285:100-102. [PubMed: 10390357]

13. Doyle DA, et al. The structure of the potassium channel: molecular basis of $\mathrm{K}^{\mathrm{b}}$ conduction and selectivity. Science. 1998; 280:69-77. [PubMed: 9525859]

14. Kuo A, et al. Crystal structure of the potassium channel KirBac1.1 in the closed state. Science. 2003; 300:1922-1926. [PubMed: 12738871]

15. Chang G, Spencer RH, Lee AT, Barclay MT, Rees DC. Structure of the MscL homolog from Mycobacterium tuberculosis: A gated mechanosensitive ion channel. Science. 1998; 282:22202226. [PubMed: 9856938]

16. Miyazawa A, Fujiyoshi Y, Unwin N. Structure and gating mechanism of the acetylcholine receptor pore. Nature. 2003; 423:949-955. [PubMed: 12827192]

17. MacKinnon R. Potassium channels and the atomic basis for selective ion conduction. Biosci Rep. 2004; 24:75-100. [PubMed: 15628664]

18. Maguire ME, Cowan JA. $\mathrm{Mg}^{2+}$ chemistry and biochemistry. Biometals. 2002; 15:203-210. [PubMed: 12206387]

19. Snavely MD, Florer JB, Miller CG, Maguire ME. Magnesium transport in Salmonella typhimurium: ${ }^{28} \mathrm{Mg}^{2+}$ transport by the CorA, MgtA, and MgtB systems. J Bacteriol. 1989; 171:4761-4766. [PubMed: 2670893]

20. Kucharski LM, Lubbe WJ, Maguire ME. Cation hexaammines are selective and potent inhibitors of the CorA magnesium transport system. J Biol Chem. 2000; 275:16767-16773. [PubMed: 10748031]

21. Szegedy MA, Maguire ME. The CorA $\mathrm{Mg}^{2+}$ transport protein of Salmonella typhimurium Mutagenesis of conserved residues in the second membrane domain. J Biol Chem. 1999; 274:36973-36979. [PubMed: 10601252]

22. Langosch D, Thomas L, Betz H. Conserved quaternary structure of ligandgated ion channels: the postsynaptic glycine receptor is a pentamer. Proc Natl Acad Sci USA. 1988; 85:7394-7398. [PubMed: 2459705]

23. Lester HA. The permeation pathway of neurotransmitter-gated ion channels. Annu Rev Biophys Biomol Struct. 1992; 21:267-292. [PubMed: 1381975]

24. Delano, WL. PyMOL. [19 October 2005] <http://pymol.sourceforge.net/>

25. Korolev S, et al. The crystal structure of spermidine synthase with a multisubstrate adduct inhibitor. Nature Struct Biol. 2002; 9:27-31. [PubMed: 11731804] 

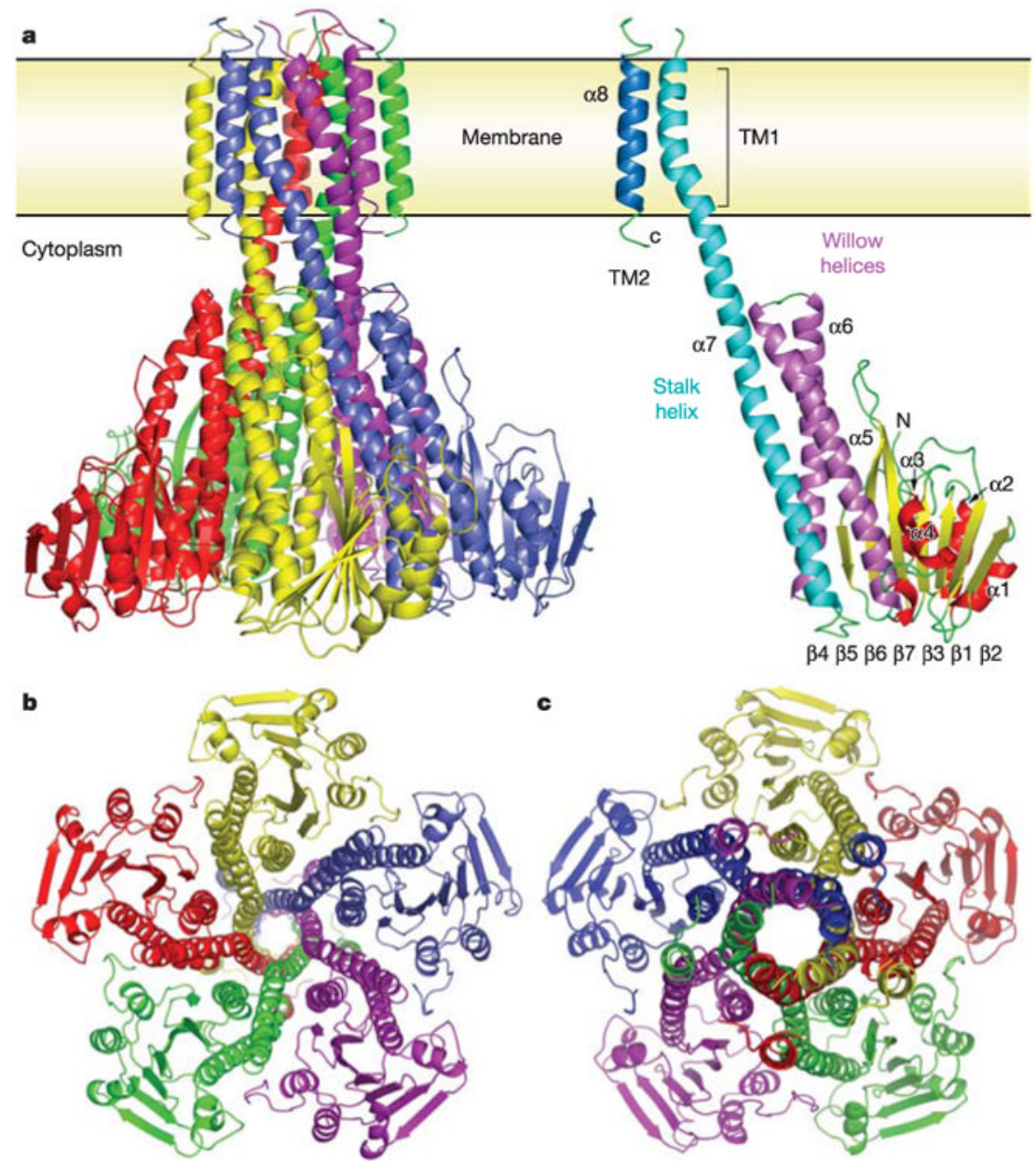

Figure 1. Structure of the CorA $\mathrm{Mg}^{2+}$ channel

a, Ribbon diagram of the CorA pentamericcomplex, viewed in the plane of the membrane. On the right is a single unit of the CorA channel highlighting the following structural features: Stalk helix and inner TM1 helix (turquoise), outer helix (dark blue) and willow helices (purple). These and the remaining $\alpha$-helices (red) and $\beta$-sheets (yellow) are numbered according to the text. The membrane surface is indicated. $\mathbf{b}$, View from the intracellular region. c, View from the periplasm. This and other figures were generated with the program PyMOL ${ }^{25}$, except where indicated. 


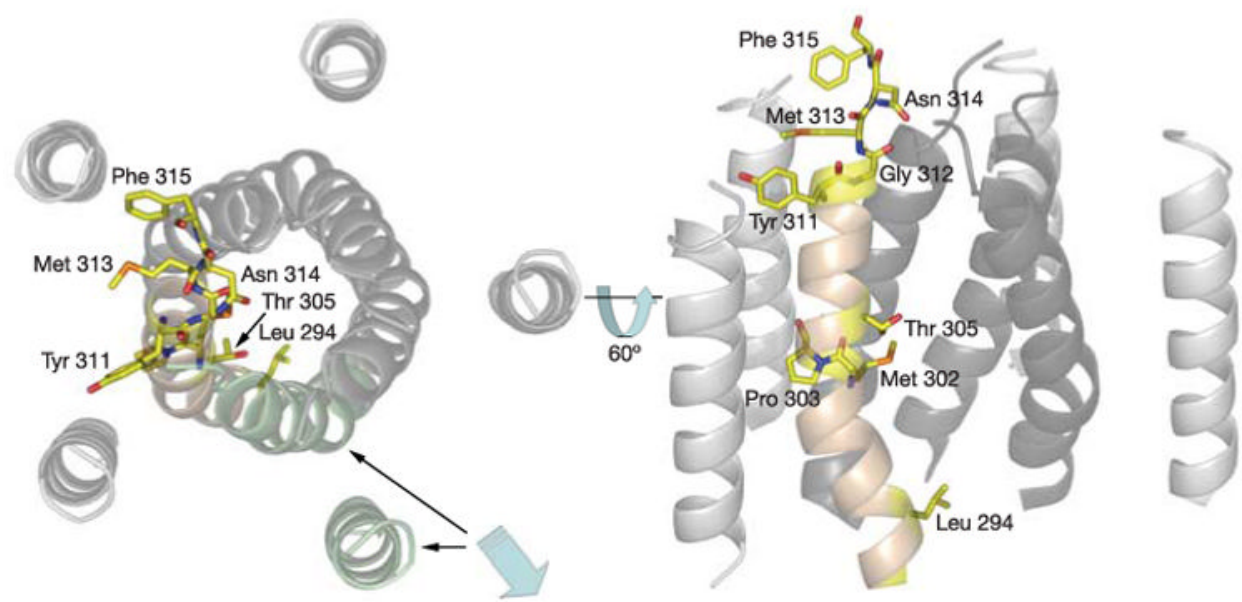

Figure 2. Analysis of the CorA pore

The CorA pore, highlighting the locations of key residues on one of the TM1 helices (pale brown), as discussed in the text. The view is looking down the pore from the periplasmic surface (left panel) and in the plane of the membrane (right panel, orientation in left panel rotated $60^{\circ}$ towards the viewer). To illustrate the pore interior better, a pair of TM1 and TM2 helices was removed (highlighted in pale green and indicated by blue arrow) for the panel on the right. 


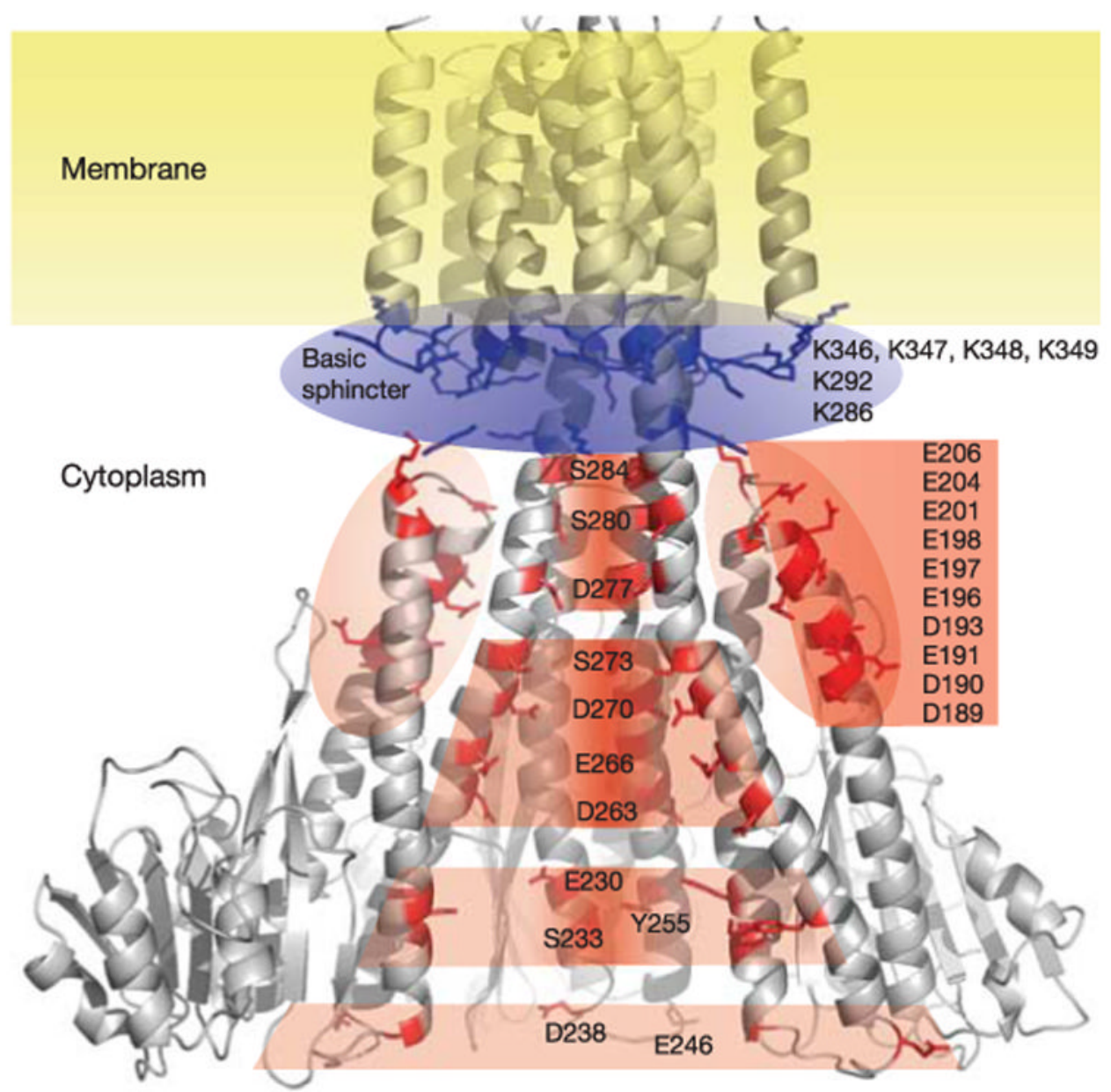

Figure 3. Electrostatic view of CorA

Positively charged residues are coloured blue and negatively charged or hydroxyl-containing residues are coloured red. Charged residues within the CorA basic sphincter (bluehighlighted region) and cytoplasmic domain (funnel interior and willow helices, redhighlighted regions) are labelled and illustrated as stick models. For better viewing of the funnel interior, two of the CorA monomers were removed from the model. 


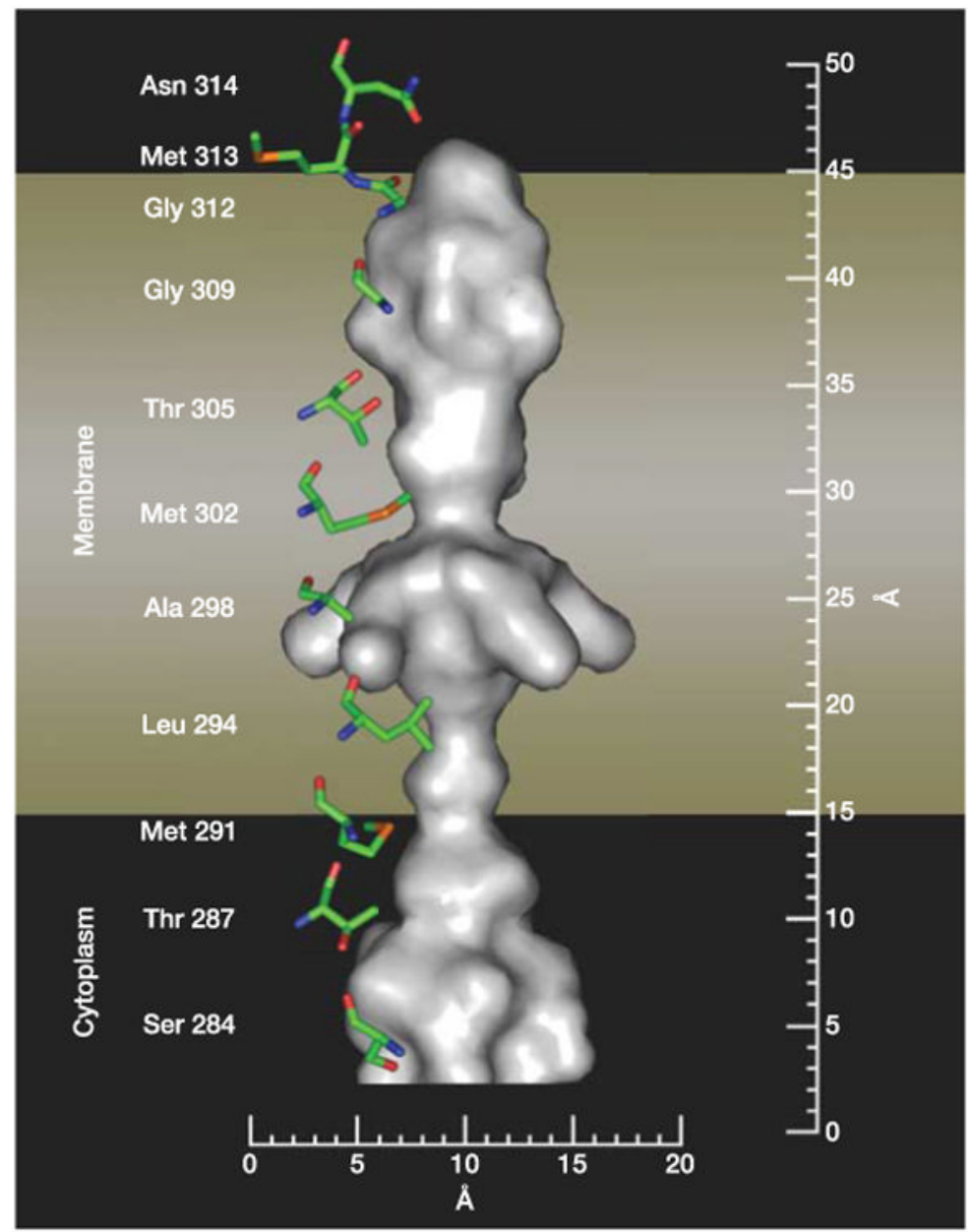

Figure 4. Pore dimensions

A cut-away view displaying the solventaccessible surface and dimensions of the CorA pore. Also shown are stick models of residues lining the pore. The key residues discussed in the text are labelled. 

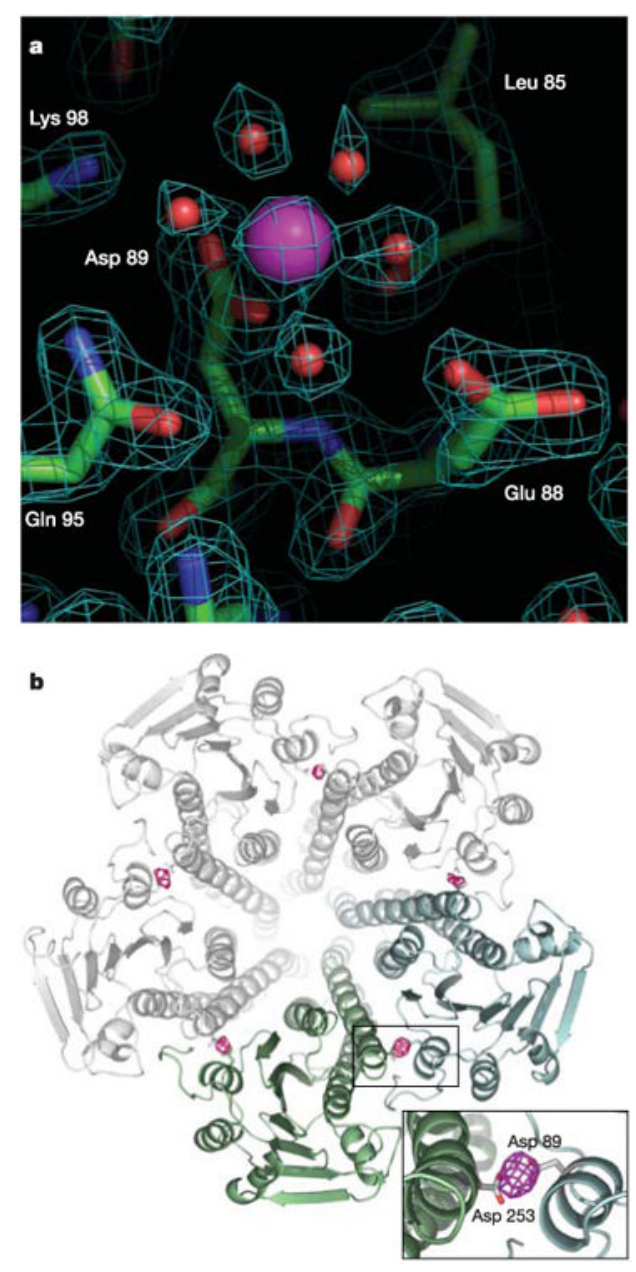

Figure 5. Electron density corresponding to protein and bound magnesium a, A portion of the soluble domain $1.85 \AA$ electron density map in the region of Asp 89, showing electron density for the putative $\mathrm{Mg}^{2+}$ ion and the water molecules that fill the hexacoordination shell. b, A portion of the $3.9 \AA$ difference Fourier electron density map (purple) showing the putative magnesium ion between monomers. The inset shows the residue Asp89 in one monomer (pale blue) and Asp253 in another monomer (green) and a peak in the difference Fourier electron density map. 